\title{
Negative group velocity of light in optical fibers
}

\author{
Miguel González Herráez*, Kwang-Yong Song and Luc Thévenaz \\ Nanophotonics and Metrology Lab. Ecole Polytechnique Federale de Lausanne, CH-1015, Switzerland \\ E-mail: miguel.gonzalez@epfl.ch. * On leave from Dept. of Electronics, Univ of Alcalá, Madrid, Spain
}

\begin{abstract}
We demonstrate experimentally that negative group velocities of light can be achieved in conventional optical fibers by use of the stimulated Brillouin scattering effect. Using the same principle, velocities as slow as $71000 \mathrm{~km} / \mathrm{s}$ can also be achieved.

(C) 2005 Optical Society of America

OCIS codes: (999.9999) Slow light; (060.4370) Nonlinear optics, fibers; (290.5900) Scattering, Brillouin
\end{abstract}

\section{Introduction}

Recent experiments have demonstrated the possibility to achieve a wide group delay control in optical fibers by use of the stimulated Brillouin scattering (SBS) effect [1,2]. Despite its recent demonstration, this technique is already thought to offer a vast potential for performing all-optical packet routing and random access memories [3]. Up to now, slow and fast light experiments in fibers using SBS have concentrated on achieving large fractional group delays in optical pulses, but with modest changes in the group velocity achieved in the fiber [1,2,4]. In this paper we demonstrate that a wide control of the group velocity of optical signals propagating in fibers can be readily achieved with conventional instrumentation. The extreme case of a negative group velocity is also demonstrated.

\section{Experimental results}

When a powerful, coherent light source tuned at a frequency $f_{0}$ is delivered into an optical fiber, two narrow bands of gain and attenuation appear in the counterpropagating sense, due to SBS, at $f_{0}-v_{B}$ and $f_{0}+v_{B}$ respectively. This sharp gain (loss) induces a strong group index change (due to the Kramers-Kronig relationships) which in turn causes a narrowband spectral region of higher (smaller) group velocity. The amount of delay (advancement) induced by the pump on a counterpropagating signal wave tuned at $f_{0}-v_{B}\left(f_{0}+v_{B}\right)$ depends linearly with the logarithmic gain (loss) achieved in the signal, with a slope of $1 \mathrm{~ns} / \mathrm{dB}$. Up to now, pump powers in the order of several milliwatts have been used in kilometer-length fibers. By the use of watt-level pump powers in meter-length fibers we have achieved similar delays to those achieved in km-long fibers (fig. 2(a)), but with much larger group index changes, as shown in Fig. 2(b). The extreme case of negative group velocity (in which the peak of the pulse exits the fiber before it enters) has also been demonstrated. The implications of this result from the point of view of the transmission of information will be discussed.

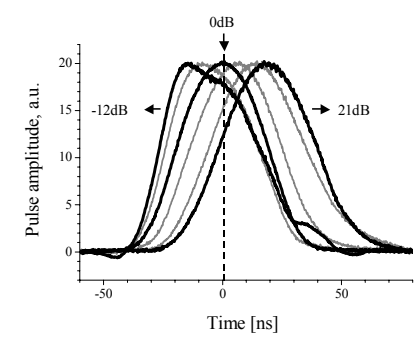

(a)

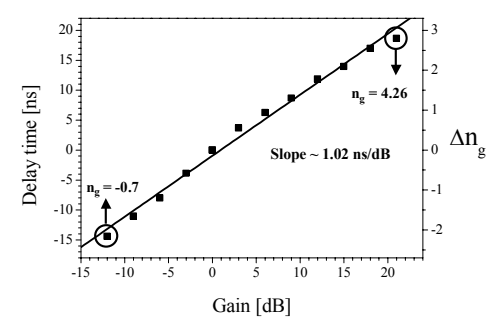

(b)

Fig. 1: (a) Pulse delaying and advancement in a 2 meter fiber using SBS (b) corresponding group delay changes. Details of the experimental setup are given in [1].

\section{References}

[1] K. Y. Song, M. G. Herráez, and L. Thévenaz, "Observation of pulse delaying and advancement in optical fibers using stimulated Brillouin scattering,” Opt. Express 13, 82-88 (2005), http://www.opticsexpress.org/abstract.cfm?URI=OPEX-13-1-82.

[2] Y. Okawachi, M.S. Bigelow, J. E. Sharping, Z. Zhu, A. Schweinsberg, D.J. Gauthier, R.W. Boyd, and A.L. Gaeta, "Tunable All-Optical Delays via Brillouin Slow Light in an Optical Fiber", Phys. Rev. Lett. 94153902 (2005).

[3] J. T. Mok and B. J. Eggleton "Expect more delays" Nature 433 811-812 (2005)

[4] K. Y. Song, M. G. Herráez, and L. Thévenaz, "Long optically-controlled delays in optical fibers" Opt. Lett. to appear (2005) 